\title{
Distributions of Unexpended Services in a D-node Markov-Renewal Network Distinguished by Customer Class
}

\author{
Richard L. Patterson \\ School of Natural Resources \\ University of Michigan \\ Ann Arbor, Michigan 48109
}

Trunsmitted by Melvin R. Scott

\begin{abstract}
Customer units from any one of $\mathrm{K}$ distinct classes arrive at random but in a fixed, known order for processing in a D-node Markov-renewal network that contains at least one absorbing node and no proper closed subsets of nodes. The joint probability function of counts of units in nodes, distinguished by customer cliss, is derived for the transient case. A numerical method for routinely computing all possible marginal distributions is demonstrated for implementation on off-the-shelf spreadhseets or database systems. The model permits explicit computation of counts by units, by class and node, for arrivals that are not Poisson distributed.

Populations of units arriving randomly in time, classes being arbitrarily mixed but in known order, represent a generic model applicable in many disciplines including biology, engineering, demography, and operations research.
\end{abstract}

\section{INTRODUCTION}

Let $(\pi(D))$ denote a family of D-node Markov-renewal networks in which each $\pi \in(\pi(D))$ contains i) no proper closed subsets of nodes, ii) at least one absorbing node, and iii) an initial node of entry into $\pi$. Having once entered $\pi$, mnits behave independently, circulating among non-absorbing nodes, until eventually absorbing nodes are entered.

Let $\Sigma(K)=\left(\sigma_{1}, \sigma_{2}, \cdots\right)$ denote a family of Poisson arrival processes where an element $\sigma_{j} \in \Sigma(K)$ is a Poisson sequence of arriving units in which exactly $K$ customer classes are represented. The order of arrivals, by 
class, is arbitrary but fixed and known. For convenience, arriving units of a given customer class are referred to by color. All $K$ colors must eventually be counted in anivals of every $\sigma \in \Sigma(K)$. A typical arrival sequence is $\sigma_{j}-$ $(R, R, G, R, R, G, \cdots) \in \Sigma(2)$. Since ordering of colors is completely arbitrary, a sequence may consist of a single color for the first $m$ arrivals, followed by arrivals of the remaining $K$-I colors.

Let $(X(t) ; t>0)$ denote a continuous time, $\mathrm{D}$-state semi-Markov process that describes internode migration of a typical arrival once it enters $\pi \in$ $\pi(D)$, independent of color. $X(t)$ is the node entered by a unit at its most recent change of node prior to time $t .(X(t))$ is characterized by an embedded Markov chain with stochastic, absorbing transition matrix $P=$ $\left(p_{i j}\right)$, conditional residence time distribution function matrix $W=\left(w_{i j}(x)\right)$, and stochastic interval transition probability function matrix $F=\left(f_{i j}(x)\right)$ $(i, j=1,2, \ldots, D)$. The element $f_{i j}(t)$ is the conditional probability that $X(t)=j$, given that the initial node (state) is $X(0+)=i$. Assuming time invariance, the conditional probability that $X(t)=j$, given that $X(z)=i$ $(0 \leqslant z<t)$ is $f_{i j}(t-z)$. Elements of $F$ are functions of elements of $P$ and $W$.

For all $\pi \in \Sigma(1)$ with arrival intensity $a(t)$ it is well known that counts of units in nodes $1,2, \ldots, D(0 \leqslant t<\infty)$ are independent Poisson distributed random variables with joint probability function

$$
\begin{aligned}
p\left(n_{1}, n_{2}, \ldots, n_{D}\right) & \equiv P\left(N_{1}(t)=n_{1}, N_{2}(t)=n_{2}, \ldots, N_{D}(t)=n_{D}\right) \\
& =\prod_{j=1}^{D} \frac{\left(m_{j}(t)\right)^{n_{j}}}{n_{j} !} e^{-m_{j}(t)}
\end{aligned}
$$

where: i) $m_{j}(t)=\int_{0}^{t} a(z) f_{j}(t-z) d z$

$$
\left(n_{j}=0,1,2, \cdots\right)(j=1, \ldots, D)
$$

Subscript $i$ denoting node of entry into $\pi$ is suppressed:

ii)

$$
\begin{aligned}
\int_{0}^{t} a(z) d z & =\int_{0}^{t} a(z) d z \sum_{j=1}^{D} f_{j}(t-z) \\
& =\sum_{j=1}^{D} \int_{0}^{t} a(z) f_{j}(t-z) d z=\sum_{j=1}^{D} m_{j}(t)
\end{aligned}
$$


Derivation of equation (1) rests upon i) the order statistic property of the Poisson process and ii) independence of movements of units upon entering $\pi$, which give rise to a multinomial model of dispersal of units in $\pi$. That is, the conditional probability of occurrence of the joint event, given $n=n_{1}$ $+\cdots+n_{D}$ units are initially in entry node $i$ at time $\approx(0 \leqq \approx<t)$, that $n_{1}, n_{2}, \ldots, n_{D}$ units are in nodes $1,2, \ldots, D$ at lime $l$ is

$$
\frac{n !}{n_{1} ! \cdots n_{D} !} f_{1}(t-z)^{n_{t}}, \ldots, f_{D}(t-z)^{n_{1}}
$$

Interval transition probabilities $f_{j}(x)$ are assumed to be positive in the interval $(0, t)$.

Our objective is to demonstrate the joint probability function $p\left(n_{1}, n_{2}, \ldots, n_{D}\right)$ for any $\pi \in \pi(L)$ and any $\sigma \in \Sigma(K)$ and, additionally, to demonstrate a numerical method for computing $p\left(n_{1}, n_{2}, \ldots, n_{D}\right)$ and any marginal probability function obtainable from it. A case study of the system $(\Sigma(2), \pi(2))$ will show how spreadsheets can be used to routinely compute $p\left(n_{1}, n_{2}, \ldots, n_{D}\right)$ and all marginal probability functions.

\section{JOINT PROBABILITIES OF COUNTS BY COLOR AND NODE}

Consider an arrival process $\sigma \in \Sigma(K)$. $\sigma$ is an infinite sequence of units where each is tagged by one of $K$ colors in an aribtrary but fixed and known order. All $K$ colors are represented. Given that $n$ arrivals have occurred in a finite interval $(0, t)$ the number of units of each color is known. Denote these numbers by $K$ indices $k_{R}(n), k_{B}(n), \ldots, k_{G}(n)$ where their sum equals $n$ for any $n=1,2, \ldots$. When the first $K-1$ indices are specified the $K$ th is determined since $k_{G}(n)=n-\sum_{j} k_{j}(n)$.

Denote counts of colored units in nodes $1,2, \ldots, D$ by random variables $N_{R 1}(t), \ldots, N_{R D}(t), \ldots, N_{G 1}(t), \ldots, N_{G D}(t)$ and let

$$
\begin{aligned}
P\left(N_{R 1}(t)=n_{R 1}, \ldots, N_{G D}(t)=n_{G D}\right) & =p\left(n_{R 1}, \ldots, n_{G D} ; t ; k_{R}, \ldots, k_{G} ; n\right) \\
& \equiv p\left(n_{R 1}, \ldots, n_{G D}\right)
\end{aligned}
$$

denote the joint probability function for occurrence of the joint event that $n_{R 1}, \ldots, n_{C D}$ units of the $K$ colors are resident in the $D$ nodes of $\pi$ at time $t$. Given that $n$ arrivals have occurred in $(0, t) p\left(n_{R 1}, \ldots, n_{G D}\right)$ factors as the product of a conditional probability $r\left(n_{R 1}, \ldots, n_{G D} ; n ; \sigma\right)$ and an uncondi- 
tional Poisson probability $q(n ; t)$ of $n$ arrivals in $(0, t)$ :

$$
p\left(n_{R 1}, \ldots, n_{G D}\right)=q(n ; t) \cdot r\left(n_{R 1}, \ldots, n_{G D} ; n ; \sigma\right)
$$

where

$$
q(n ; t)=\frac{m(t)^{n}}{n !} e^{-m(t)} ;\left(m(t)=\int_{0}^{t} a(x) d x\right)
$$

and

$$
\begin{aligned}
& r\left(n_{R 1}, \ldots, n_{G D} ; n ; \sigma\right) \\
& \quad=\left(\frac{k_{R} !}{n_{R 1} ! \ldots n_{R D} !} \cdot \prod_{j=1}^{D} \alpha_{j}^{n_{n_{i}}}\right) \times \ldots \times\left(\frac{k_{G} !}{n_{G !} ! \cdots n_{G D} !} \cdot \prod_{j=1}^{n} \alpha_{j}^{n_{(; j}}\right)
\end{aligned}
$$

Probabilities $\alpha_{j} \equiv \alpha_{j}(t)$ are equal to

$$
\alpha_{j}=\frac{m_{j}(t)}{m(t)}(j=1, \ldots, D)
$$

The right side of Equation (3) is defined to be zero for all vectors $\left(n_{R l}, \ldots, n_{R D}, \ldots, n_{G l}, \ldots, n_{G D}\right)$ not satisfying required conditions on the $n_{i j}$ 's, namely:

$$
\begin{gathered}
n_{i j}=0,1,2, \ldots ; i=R, B, \ldots, G ; j=1,2, \ldots, D \\
n_{R 1}+\cdots+n_{R D}=k_{R} ; \ldots ; n_{G 1}+\cdots+n_{G D}=k_{G} \\
k_{R}+\cdots+k_{G}=n .
\end{gathered}
$$

Equation (3) defines a proper probability distribution containing a total of

$$
\left(\begin{array}{c}
k_{R}+D-1 \\
k_{R}
\end{array}\right) \times \cdots \times\left(\begin{array}{c}
k_{G}+D-1 \\
k_{G}
\end{array}\right)
$$

multinomial terms which is the number of distinct non-negative integer vectors $\left(n_{R l}, \ldots, n_{R D}, \ldots, n_{G l}, \ldots, n_{G D}\right)$ satisfying the constraints given above. 
The probabilities $\alpha_{1}, \ldots, \alpha_{D}$ are assumed to be color-independent. If not, Equation (3) holds after indexing the $\alpha$ 's on color as well as node. Even when they are color-independent node-count probabilities are not "colorblind". That is, if a pair of units of different colors are swapped between two nodes with node count totals holding constant, the joint probabilities of the two vectors may be unequal. To show this, multiply and divide Equation (3) by the product

$$
\left(n_{R 1}+\cdots+n_{G 1}\right) ! \times \cdots \times\left(n_{R D}+\cdots+n_{G D}\right) !
$$

Then rearranging and combining terms we have

$$
\begin{aligned}
& p\left(n_{R 1}, \ldots, n_{R D}, \ldots, n_{G 1}, \ldots, n_{G D}\right) \\
& =\prod_{j=1}^{D} \frac{m_{j}(t)^{n_{j}}}{n_{j} !} e^{-m_{j}(t)} \cdot\left\{\frac{\left(\begin{array}{c}
n_{1} \\
\left.n_{R 1}, \ldots, n_{G 1}\right) \times \cdots \times\left(\begin{array}{c}
n_{D} \\
n_{R D}, \ldots, n_{G D}
\end{array}\right)
\end{array}\right\}}{\left(k_{R}, \ldots, k_{G}\right)}\right\} \\
& =\prod_{j=1}^{D} \frac{m_{j}(t)^{n_{i}}}{n !} e^{-m_{j}(t)} \cdot\left\{\frac{\left(\begin{array}{c}
k_{R} \\
n_{R 1}, \ldots, n_{R D}
\end{array}\right) \times \cdots \times\left(\begin{array}{c}
k_{G} \\
n_{G 1}, \ldots, n_{G D}
\end{array}\right)}{\left(n_{1}, \ldots, n_{D}\right)}\right\}
\end{aligned}
$$

where $n=n_{1}+n_{n_{j}}+\cdots+n_{D} ; n_{j}=n_{R j}+\cdots+n_{G j}(j=1, \ldots, D)$; and quantities $\left(n_{R j}, \ldots, n_{G j}\right)$ are multinomial coefficients.

The terms that vary, causing changes in the right hand side of equation (4) are the products

$$
\prod_{j=1}^{D} n_{R j} ! \cdots n_{G j} !
$$

All other terms on the right side of (4) remain unchanged when pairs of different colored units are swapped between nodes.

Equation (4) also illustrates another argument for deducing the joint probability function $p\left(n_{R 1}, \ldots, n_{G D}\right)$ whenever the probabilities $\alpha_{j}$ are color independent. Both Equations (3) and (4) reduce to equation (1) if colors are 
not distinguished. The right hand side of equation (4) may also be written in terms of binomial coefficients.

Equation (4) shows that the joint probability function factors as the product of two functions, the first depending on network topology and the second depending on the ordering of colors. The right side of (4) also indicates the degree to which dependence occurs among random variables $N_{i j}$. If the ratios of multinomial coefficients were all equal to one, mutual independence would hold as in Equation (1).

Distinct arrival processes $\sigma_{i}$ and $\sigma_{j}$ give rise to distinct probability distributions on the sample space of vectors $\left(n_{\lambda 1}, \ldots, n_{C_{i} D}\right)$. Differences may or may not be recognized in numerical computations because the processes may differ only beyond some large $n$ whose Poisson probabilities match for numbers of arrivals less than $n$.

\section{NON-POISSON ARRIVAL PROCESSES}

Non-Poisson arrival processes are created by treating units of certain colors as position markers only, where marginal distributions of counts of other colors reflect non-Poisson distributed arrivals of those colors. For example, consider an arrival sequence $\sigma \in \Sigma(2)$ where $\sigma=(R, B, R, B, \cdots)$ consists of arrivals alternating between two colors $R$ and $B$. Marginal probabilities $p\left(n_{R 1}, \ldots, n_{R D}\right)$ ignoring units of color $B$ reflect arrivals of color $R$ which are generated by a renewal process where interarrival intervals are Gamma distributed. Other arrival processes which are neither Poisson nor of the renewal type can be created by sequences such as $\sigma \in \Sigma(3)$ where $\sigma=(R, B, G, R, B, B, G, \ldots)$ for which marginal probabilities of counts of color $G, R$, or $B$ all reflect arrival processes whose interarrival times are independent but not identically distributed.

\section{COMPUTATION OF JOINT AND MARGINAL PROBABILITIES}

Marginal probabilities are obtained by summing over subsets of probabilities corresponding to vectors in the sample space of the random vector $\left(N_{R 1}, \ldots, N_{G 1}, \ldots, N_{R D}, \ldots, N_{G D}\right)$ in which conditions on subsets of columns are fixed in advance. Computations can be performed in two ways. In the first method, joint probabilities are computed from either Equations (3) or (4), in which columns of the random vector correspond to fields in a data base. Individual sample vectors correspond to records. Marginal probabilities are simply tabulated by summing over appropriate subsets of columns. The advantage of this method is its simplicity of implementation and the ability to compute every possible marginal probability distribution routinely and with- 
out danger of committing errors in formulae preparation. The disadvantage is that superfluous joint probabilities may have to be computed for a given problem. Additionally, the method becomes impractical for problems in which very large numbers of sample vectors are involved. This technique is demonstrated in the case study that follows.

The second method for computing marginal probabilities consists of writing formulae that compute and sum joint probabilities from Equations (3) or (4) over subsets of sample vectors, using standard theory for formulate creation. The advantages and disadvantages of this method are reversed from those of the first method. A factor that complicates formulae creation is the presence of the indices $k_{j}$.

Considerations that apply to cither method are i) number of sample vectors for which probabilities must be computed, and ii) speed of computation. The first consideration has two aspects which are 1) necessary and sufficient numbers of sample vectors for which probabilities must be computed and 2) required error bounds on computation on sums of probabilities and on approximation of individual probabilities. The second factor relates to the first inasmuch as numbers of sample vectors and error bounds affect speed of computations. Additionally, performance of code that computes and sums probabilities over subsets of sample vectors is another aspect of overall speed of computation. A tradeoff exists between methods in this respect. This paper does not provide definitive answers to all of the above considerations. Some answers are given, however. Equation (3) is used to compute a sufficient number of sample vectors whose joint probabilities must be computed. The probability function $q(n ; t)$ is summed from $n=0$ to $n=n(\max )$, where $n(\max )$ is to be determined as follows:

i) Set the time $t$ and evaluate $m(t)$;

ii) Select a value $\varepsilon(0<\varepsilon \ll 1)$ for which the sum of Poisson probabilities $q(n ; t)$ from $n=0$ through $n=n(\max )$ must be at least as large as $1-\varepsilon$;

iii) Find the smallest $n(\max )$ for which

$$
\sum_{n=0}^{n(\max )} \frac{m(t)^{n}}{n !} e^{-m(t)} \geqq 1-\varepsilon ;(n(\max )=0,1,2, \ldots) ;
$$

iv) Compute the required number of sample vecturs whose joint probalsilities must be computed by the formula

Required Number

$$
=1+\sum_{n=1}^{n(\max )}\left\{\left(\begin{array}{c}
k_{R}(n)+D-1 \\
k_{R}(n)
\end{array}\right) \times \cdots \times\left(\begin{array}{c}
k_{G}(n)+D-1 \\
k_{G}(n)
\end{array}\right)\right\}
$$


The term $r\left(n_{R 1}, \ldots, n_{G D} ; n ; \sigma\right)$ on the right hand side of equation (3) distributes the probability mass $q(n ; t)$ over a number of sample vectors given by the product of multinomial probabilities inside the brackets on the right side of Equation (5). In order for the sum of joint probabilities to sum to at least $1-\varepsilon$, care must be exercised in determining the approximation error acceptable in computing the joint probabilities that must be summed. Premature roundoff of significant digits leads to a sum of probabilities that is less than $1-\varepsilon$. As the number of sample vectors increases, the danger of incorrect approximation of individual joint probabilities increases.

Equation (5) gives the least upper bound on the number of sample vectors whose joint probabilities must be computed. Some sample vectors may be included whose probabilities are smaller than the minimum required by the limit of roundoff error. Those probabilities are, in effect, set equal to zero, and time required to compute them is wasted. If they can be identified in advance they may be excluded from the "required number" computed by Equation (5). The set of probabilities and associated vectors obtained by reducing the set implied by Equation (5) by the subset just identified gives a minimal sufficient set of sample vectors from which all marginal distributions can be computed. When the specific marginal distributions are given that must be computed, the minimal sufficient set is further reduced, which determines a necessary and sufficient set whose joint probabilities must be computed for the given marginal distribution. The set of sample vectors that are necessary and sufficient for computation of a specific marginal distribution can, in general, only be determined by the second method of computation given above.

To illustrate points outlined above, consider an example of a network $\pi \subset \pi(2)$ and an arrival process $\sigma \in \Sigma(2)$ where $\sigma=(R, G, R, G, \ldots)$. Arriving units enter node 1 initially and after a random time in residence, enter a sink node 2. Let $a(t)=1$ and let $t=1$. Suppose a number of sample vector probabilities must be computed sufficient for their sum to bc within $\varepsilon=0.005$ of unity. From a table of Poisson probabilities, the smallest $n$ from which the sum of Poisson probabilities, from 0 through $n$ is $n=n(\max )=4$. Entering Table 1a for $D=2$ and $n=4$, the number of vector probabilitics that must be computed is no greater than the sum of entries in the $D=2$ column from $n=1$ through $n=4$, plus 1 for the case $n=0$, or a total of 22 . If the allowable deviation from unity is set at $\varepsilon=0.001$, the number of vectors for which probabilities must be computed is at most 34 . Tables $2 \mathrm{a}$ and $2 \mathrm{~b}$ show the nine vector probabilities for $n=4$ when mean residence time in node 1 is 1.0 and 0.1 , respectively. In Table $2 \mathrm{a}$, all but the single starred probability must be computed for the sum to reach 0.015 , which is the difference between the sum $0.981(n=3)$ and the sum $0.996(n=4)$. In Table $2 \mathrm{~b}$, only the four non-starred probabilities need to be computed for the 
TABLE 1A

\begin{tabular}{rccrrrrrr}
\multicolumn{8}{c}{ NUMBER OF SAMPLE VECTORS FOR $\sigma=(R, G, R, G, \ldots) ; K=2$} \\
\hline$n$ & $k_{R}$ & $k_{G}$ & 2 & 3 & 4 & 5 & 6 \\
\hline $\mathbf{I}$ & 1 & 0 & 2 & 3 & 4 & 5 & 6 \\
2 & 1 & 1 & 4 & 9 & 16 & 25 & 36 \\
3 & 2 & 1 & 6 & 18 & 40 & 75 & $\ldots$ \\
4 & 2 & 2 & 9 & 36 & 100 & $\cdots$ & $\ldots$ \\
5 & 3 & 2 & 12 & 60 & 200 & $\ldots$ & $\ldots$ \\
6 & 3 & 3 & 16 & 100 & 400 & $\cdots$ & $\ldots$ \\
$\vdots$ & & & & & & & \\
\hline
\end{tabular}

TABLE 1B

NUMBER OF SAMPLE VECTORS FOR $\sigma=(R, G, B, R, G, B, \ldots) ; K=3$

$\mathrm{D}$

\begin{tabular}{rrrrrrrrr}
$n$ & $k_{R}$ & $k_{C}$ & $k_{B}$ & 2 & 3 & 4 & 5 & 6 \\
\hline 1 & 1 & 0 & 0 & 2 & 3 & 4 & 5 & 6 \\
2 & 1 & 1 & 0 & 4 & 9 & 16 & 25 & 36 \\
3 & 1 & 1 & 1 & 8 & 27 & 64 & 125 & $\cdots$ \\
4 & 2 & 1 & 1 & 12 & 54 & 160 & $\cdots$ & \\
5 & 2 & 2 & 1 & 18 & 108 & 400 & $\cdots$ & \\
6 & 2 & 2 & 2 & 27 & 216 & 1000 & $\cdots$ &
\end{tabular}

TABLE 1C

NUMBER OF SAMPLE VECTORS FOR $\sigma=(R, G, B, W, R, G, B, W, \ldots) ; K=4$

\begin{tabular}{rrrrrrrrrr}
\hline$n$ & $k_{R}$ & $k_{G}$ & $k_{B}$ & $k_{W}$ & 2 & 3 & 4 & 5 & 6 \\
\hline 1 & 1 & 0 & 0 & 0 & 2 & 3 & 4 & 5 & 6 \\
2 & 1 & 1 & 0 & 0 & 4 & 9 & 16 & 25 & 36 \\
3 & 1 & 1 & 1 & 0 & 8 & 27 & 64 & 125 & $\cdots$ \\
4 & 1 & 1 & 1 & 1 & 16 & 81 & 256 & 625 & $\cdots$ \\
5 & 2 & 1 & 1 & 1 & 24 & 162 & 640 & $\cdots$ & \\
6 & 2 & 2 & 1 & 1 & 36 & 324 & 1600 & $\ldots$ & \\
$\vdots$ & & & & & & & & & \\
\hline
\end{tabular}


TABLE 2A

PROBABILITIES OF VECTORS FOR THE CASE $K=2 ; n=4$; $a t=1$; NODE 1 MEAN RESIDENCE TIME $=1$

\begin{tabular}{ccccc}
\hline$n_{R 1}$ & $n_{R 2}$ & $n_{G 1}$ & $n_{G 2}$ & $p\left(n_{R 1}, n_{R 2}, n_{G 1}, n_{C 2}\right)$ \\
\hline 2 & 0 & 2 & 0 & 0.002447337912 \\
2 & 0 & 1 & 1 & 0.002848587317 \\
2 & 0 & 0 & 2 & 0.000828905733 \\
1 & 1 & 2 & 0 & 0.002848587317 \\
1 & 1 & 1 & 1 & 0.0033156229332 \\
1 & 1 & 0 & 2 & 0.000964807657 \\
0 & 2 & 2 & 0 & 0.000828905733 \\
0 & 2 & 1 & 1 & 0.000964807657 \\
0 & 2 & 0 & 2 & $0.000280747792 *$ \\
& & & Sum & 0.0153283310050 \\
\hline
\end{tabular}

TABLE $2 \mathrm{~B}$

PROBABILITIFS OF VECTORS FOR THE CASE, $K=2 ; n=4$; $a t=1$; NODE 1 MEAN RESIDENCE TIME $=0.1$

\begin{tabular}{ccccc}
\hline$n_{R 1}$ & $n_{R 2}$ & $n_{G 1}$ & $n_{G 2}$ & $p\left(n_{R 1}, n_{R 2}, n_{G 1}, n_{G 2}\right)$ \\
\hline 2 & 0 & 2 & 0 & $0.000001532^{*}$ \\
2 & 0 & 1 & 1 & $0.000027587^{*}$ \\
2 & 0 & 0 & 2 & $0.000124149^{*}$ \\
1 & 1 & 2 & 0 & $0.000027587^{*}$ \\
1 & 1 & 1 & 1 & 0.000496597 \\
1 & 1 & 0 & 2 & 0.002234799 \\
0 & 2 & 2 & 0 & $0.000124149^{*}$ \\
0 & 2 & 1 & 1 & 0.002234799 \\
0 & 2 & 0 & 2 & 0.010057107 \\
& & & Sum & 0.015328296 \\
\hline
\end{tabular}

rounded sum to reach 0.015 . A savings of 55 percent in the number of probabilities that need to be computed would have been achieved while meeting the error margin requirement if it could have been efficiently determined in advance i) whether significant savings in computation time would have been achieved and ii) which vector probabilities need not have been computed. As $\varepsilon$ diminishes, larger values of $n(\max )$ are required, which leads to a nonlinear increase in the number of probabilities that potentially must be computed. Computation time will increase exponentially as $n(\max )$ increases due to the factorials present in Equation (5) unless a recursive 
method for their computation is employed. Even for small systems such as the one demonstrated in the case study below, recursive methods for building tables of probabilities yield significant savings in computation time when spreadsheet or database languages (method 1), inherently slow, are employed for computations of distributions.

\section{CASE STUDY FOR A SYSTEM IN (П(2), $\Sigma(2))$}

Let $\sigma \in \mathbf{\Sigma}(2)$ be the sequence $\sigma=(R, B, R, B, \ldots)$ of Poisson distributed arrivals with constant arrival intensity $a$. Let $\pi \in \pi(2)$ be a two-node network in which arrivals enter node 1 , whereupon after random, independent times in residence in node 1 , they enter absorbing node 2 . Residence time cdf in node 1 is assumed to have the form $F(x)=1-e^{-b x}(b>0)$. This is the simplest of all topologies that can be defined for two node networks. Interval transition probability functions are $f_{1}(t)=1-F(t)$ and $f_{2}(t)=F(t)$. Functions $m_{1}(t), m_{2}(t)$ and $m(t)$ are

$$
\begin{gathered}
m_{1}(t)=a \cdot \int_{0}^{t}(1-F(x)) d x=\frac{a}{b}\left(1-e^{-b \prime}\right) \\
m_{2}(t)=a \cdot \int_{0}^{t} F(x) d x=a \cdot t-\frac{a}{b}\left(1-e^{-b t}\right) ; \\
m(t)=m_{1}(t)+m_{9}(t)=a \cdot t
\end{gathered}
$$

Let $\varepsilon=5.0 \times 10^{-6}, a=1, b=1$, and $t=1$. Then $n(\max )=7$. From Equation (5) the maximum number of sample vectors for which probabilities must be computed is 70 . Individual joint probabilities are computed from equation (4) and listed in Table 3 under the heading $p\left(n_{R 1}, n_{R 2}, n_{G 1}, n_{G 2}\right)$. For $n=7$ all twenty probabilities are listed as 0.0000 although when summed they contribute a rounded total of 0.0001 to the accumulated sum of probabilitics. Had they becn carried as having value zero, their contribution to the accumulated sum would have been zero.

Marginal probabilities are tabulated routinely from Table 3. The marginal probability function $p\left(n_{R 1}, n_{G 1}\right)$ illustrated in Table 4 is obtained by summing $p\left(n_{R 1}, n_{G 1}, n_{R 2}, n_{G 2}\right)$ over all values of $n_{R_{2}}$ and $n_{G 2}$. A roundoff error of 0.0002 is created by summing only over the first four unrounded digits as shown in the Table. The marginal probability function $p\left(n_{R 1}, n_{R 2}\right)$ depicted 
TABLE 3

JOINT PBOBABILITY FLNCTION FOR THE CASE $K=2 ; t=1$; $a t=1$; NODE 1 MFAN RESIIDFNCE TIMF, $=1.0$

\begin{tabular}{|c|c|c|c|c|c|c|c|c|c|c|c|}
\hline time:t & $\begin{array}{c}\text { no. } \\
\text { atriji:n }\end{array}$ & $n r l$ & $n r^{2}$ & 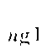 & $n_{n} 2$ & $K r !$ & $n r 2 !$ & $\operatorname{mat} 2 !$ & $n-k r !$ & $\begin{array}{l}\text { arriv } \\
\text { rate }\end{array}$ & $\begin{array}{l}\text { ress } \\
\text { time inv }\end{array}$ \\
\hline 1 & 0 & 0 & 0 & 0 & 0 & ] & 1 & 1 & 1 & 1. & 1 \\
\hline 1 & 1 & l & 0 & 0 & 0 & 1 & 1 & 1 & 1 & 1 & 1 \\
\hline 1 & & 0 & I & 0 & (I) & 1 & 1 & 1 & 1 & 1 & 1 \\
\hline 1 & 2 & 1 & 0 & 1 & 1) & 1 & 1 & 1 & 1 & $\mathrm{l}$ & 1 \\
\hline 1 & & 1 & 0 & 0 & $\mathbf{I}$ & 1 & 1 & l & 1 & 1. & 1 \\
\hline 1 & & 0 & 1 & 1 & 0 & 1 & i & i & 1 & 1 & 1 \\
\hline 1 & & 0 & 1 & 0 & 1 & 1 & 1 & 1 & 1 & 1 & 1 \\
\hline 1 & 3 & 2 & 0 & 1 & () & 2 & 1. & 1 & 1 & 1 & 1 \\
\hline 1 & & 2 & 0 & 0 & 1 & 2 & 1 & 1 & 1 & 1 & 1 \\
\hline 1 & & 1 & 1 & 1 & 0 & 2 & 1 & 1 & l & 1 & 1 \\
\hline 1 & & 1 & 1 & 0 & 1 & 2 & 1 & 1 & 1 & 1. & 1 \\
\hline 1. & & 0 & 2 & 1 & 0 & 2 & 2 & 1. & 1 & 1 & 1 \\
\hline 1 & & 0 & 2 & 0 & 1 & 2 & 2 & 1 & 1 & 1 & 1 \\
\hline l & 4 & 2 & 0 & 2 & 0 & $\overline{2}$ & 1 & 1 & 2 & i & 1 \\
\hline 1 & & 2 & 0 & 1 & 1 & 2 & 1 & 1. & 2 & 1. & 1 \\
\hline 1 & & 2 & 0) & 0 & 2 & 2 & 1 & 2 & 2 & 1 & 1 \\
\hline 1 & & 1 & l & 2 & 0 & 2 & l & 1 & 2 & 1 & 1 \\
\hline 1 & & 1 & 1 & 1 & 1 & 2 & 1 & 1 & 2 & 1 & $\mathbf{1}$ \\
\hline 1 & & 1 & 1 & 0 & 2 & 2 & 1 & 2 & 2 & 1 & 1 \\
\hline l & & 0 & 2 & 2 & 0 & 2 & 2 & 1 & 2 & 1 & 1 \\
\hline 1 & & 0 & 2 & 1 & 1 & 2 & 2 & 1 & 2 & 1 & 1 \\
\hline 1 & & 0 & 2 & 0 & 2 & 2 & 2 & 2 & 2 & 1 & 1 \\
\hline J. & 5 & 3 & 0 & 2 & 0 & 6 & 1 & ] & 2 & 1 & $\mathbf{I}$ \\
\hline 1 & & 3 & 0 & 1 & 1 & 6 & 1 & 1 & 2 & 1 & 1 \\
\hline 1 & & 3 & 0 & 0 & 2 & 6 & 1 & 2 & 2 & 1 & 1 \\
\hline 1 & & 2 & 1 & 2 & 0 & 6 & ] & 1 & 2 & 1 & 1 \\
\hline 1 & & 2 & 1 & 1 & 1 & 6 & 1 & 1 & 2 & 1 & 1 \\
\hline 1 & & 2 & 1 & 0 & 2 & 6 & 1 & 2 & 2 & 1 & 1 \\
\hline 1 & & 1 & 2 & $\underline{2}$ & is & 6 & 2 & 1 & $\lambda$ & 1 & 1 \\
\hline 1 & & 1 & 2 & 1 & 1 & 6 & 2 & 1 & 2 & 1. & 1 \\
\hline l & & 1 & 2 & 0 & 2 & 6 & 2 & 2 & 2 & 1 & 1 \\
\hline 1 & & 0 & 3 & 2 & 0 & 6 & 6 & 1 & 2 & 1 & 1 \\
\hline 1 & & 0 & 3 & 1 & 1 & 6 & 6 & 1 & 2 & 1 & 1 \\
\hline 1 & & 0 & 3 & 0 & 2 & 6 & 6 & 2 & 2 & 1 & 1 \\
\hline l & 6 & 3 & 0 & 3 & 0 & 6 & 1 & 1 & 6 & 1 & 1 \\
\hline 1 & & 3 & 0 & 2 & 1 & 6 & 1 & l & 6 & 1 & I \\
\hline 1 & & 3 & 0 & 1 & 2 & 6 & 1 & 2 & 6 & 1 & 1 \\
\hline 1 & & 3 & 0 & 0 & 3 & 6 & 1 & 6 & 6 & $\mathrm{l}$ & 1 \\
\hline 1 & & 2 & 1 & 3 & 0 & 6 & 1 & 1 & 6 & 1 & 1 \\
\hline 1 & & 2 & 1 & 2 & 1 & 6 & 1 & 1 & 6 & 1 & 1 \\
\hline 1 & & 2 & 1 & 1 & 2 & 6 & 1 & 2 & 6 & 1 & 1 \\
\hline 1 & & 2 & 1 & 0 & 3 & 6 & 1 & 6 & 6 & 1 & 1 \\
\hline 1 & & 1 & 2 & 3 & () & 6 & 2 & 1 & 6 & 1 & 1 \\
\hline 1 & & 1 & 2 & 2 & 1 & 6 & 2 & 1 & 6 & I & 1 \\
\hline 1 & & 1 & $\bar{z}$ & I. & 2 & 6 & 2 & 2 & 6 & 1 & 1 \\
\hline 1 & & 1 & 2 & 0 & 3 & 6 & 2 & 6 & 6 & 1 & 1 \\
\hline 1 & & 0 & 3 & 3 & 0 & 6 & 6 & 1. & 6 & l & 1 \\
\hline 1 & & 0 & 3 & 2 & 1 & 6 & 6 & 1 & 6 & 1 & 1 \\
\hline 1 & & 0 & 3 & 1 & 2 & 6 & 6 & 2 & 6 & 1 & 1 \\
\hline 1 & & 0 & 3 & 0 & 3 & b & 6 & 6 & G & 1 & 1 \\
\hline 1 & $\vec{i}$ & 4 & 0 & 3 & 0 & 24 & 1 & 1 & 6 & 1 & 1 \\
\hline 1 & & 4 & 0 & 2 & 1 & 24 & 1 & 1 & 6 & l & 1 \\
\hline 1 & & 4 & 0 & 1 & 2 & 24 & 1 & 2 & 6 & 1 & 1 \\
\hline 1 & & 4 & 0 & 0 & 3 & 24 & 1 & 6 & 6 & 1 & 1 \\
\hline 1. & & 3 & 1 & 3 & 0 & 24 & 1 & 1 & 6 & 1 & 1 \\
\hline 1 & & 3 & 1 & 2 & 1 & 24 & 1 & 1 & 6 & 1 & 1 \\
\hline 1 & & 3 & 1 & 1 & 2 & 24 & 1. & 2 & 6 & 1 & 1 \\
\hline 1 & & 3 & I & 0 & 3 & 24 & 1 & 6 & 6 & 1 & 1 \\
\hline 1 & & 2 & 2 & 3 & 0 & 24 & 2 & 1 & 6 & 1 & 1 \\
\hline ] & & 2 & 2 & 2 & 1 & 24 & 2 & 1 & 6 & 1 & 1 \\
\hline 1 & & 2 & 2 & 1 & 2 & 24 & 2 & 2 & 6 & 1 & 1 \\
\hline 1 & & 2 & 2 & 0 & 3 & 24 & 2 & 6 & 6 & 1 & 1 \\
\hline 1 & & 1 & 3 & 3 & 0 & 24 & 6 & 1 & 6 & 1 & 1 \\
\hline 1 & & 1 & 3 & 2 & 1. & 24 & 6 & l & 6 & 1 & 1 \\
\hline 1 & & 1 & 3 & 1 & 2 & 24 & 6 & 2 & 6 & 1 & 1 \\
\hline 1 & & 1 & 3 & 0 & 3 & 24 & 6 & 6 & 6 & 1 & 1 \\
\hline 1 & & 0 & 4 & 3 & 0 & 24 & 24 & 1 & 6 & 1 & 1 \\
\hline l & & 0 & 4 & 2 & 1 & 24 & 24 & 1 & 6 & 1 & 1 \\
\hline 1 & & 0 & 4 & 1 & 2 & 24 & 24 & 2 & 6 & 1 & 1 \\
\hline 1 & & 0 & 4 & 0 & 3 & 24 & 24 & 6 & 6 & 1 & 1 \\
\hline
\end{tabular}


TABLE 3-(Continued)

\begin{tabular}{|c|c|c|c|c|c|c|c|c|}
\hline$n !$ & $\begin{array}{c}n r]+ \\
n g l !\end{array}$ & $\begin{array}{c}n r 2+ \\
n g 2 !\end{array}$ & $n r 1 !$ & ng 1 !! & $\begin{array}{c}p(n+1, n+2 . \\
n g 1, n g 2)\end{array}$ & $p(n: a t-1)$ & $\begin{array}{l}\text { cum } \\
\text { prob }\end{array}$ & weights \\
\hline 1 & 1 & 1 & 1 & 1 & 0.3679 & 0.3679 & 0.3679 & 1.0000 \\
\hline 1 & 1 & 1 & 1 & 1 & 0.2325 & & & 1.0000 \\
\hline 1 & 1 & 1 & 1 & 1 & 0.1353 & 0.3679 & 0.7358 & 1.00000 \\
\hline 2 & 2 & 1 & 1 & 1 & 0.0735 & & & 1.00000 \\
\hline 2 & 1 & 1 & j & 1 & 0.0428 & & & 0.5000 \\
\hline 2 & 1 & 1 & 1 & 1 & $0.1) 428$ & & & 0.5000 \\
\hline 2 & 1 & 2 & 1 & 1 & 0.0249 & 0.1839 & 0.9197 & 1.0000 \\
\hline 6 & 6 & 1. & 2 & 1 & 0.015 .5 & & & 1.0000 \\
\hline 6 & 2 & 1 & 2 & 1 & 0.0090 & & & 0.3333 \\
\hline 6 & 2 & 1 & 1 & 1 & 0.0180 & & & 0.6667 \\
\hline 6 & 1 & 2 & 1 & 1 & 0.0105 & & & 0.6667 \\
\hline 6 & 1 & 2 & 1 & 1 & 0.0052 & & & 0.3333 \\
\hline 6 & 1 & 6 & 1 & 1 & 0.0031 & 0.0613 & 0.9810 & 1.0000 \\
\hline 24 & 24 & 1 & 2 & 2 & 0.0024 & & & 1.0000 \\
\hline 24 & 6 & 1 & 2 & 1 & 0.0028 & & & 0.5000 \\
\hline 24 & 2 & 2 & 2 & 1 & 0.0008 & & & 0.1667 \\
\hline 24 & 6 & 1 & ] & 2 & 0.0028 & & & 0.5000 \\
\hline 24 & 2 & 2 & 1 & 1 & 0.00033 & & & 0.66677 \\
\hline 24 & 1 & 6 & 1 & 1 & 0.0010 & & & 0.5000 \\
\hline 24 & 2 & 2 & I & 2 & 0.0006 & & & 0.1667 \\
\hline 24 & 1 & 6 & 1 & 1 & 0.0010 & & & 0.5000 \\
\hline 24 & 1 & 24 & 1 & 1 & 0.0003 & 0.153 & 0.9963 & 1.0000 \\
\hline 1.20 & 120 & 1 & 6 & 2 & 0.0003 & & & 1.0000 \\
\hline 120 & 24 & 1 & 6 & 1 & 0.0004 & & & 0.4000 \\
\hline 120 & 6 & 2 & 6 & 1. & 0.0001 & & & 0.1000 \\
\hline 120 & 24 & 1 & 2 & 2 & 0.000 .5 & & & 0.6000 \\
\hline 120 & 6 & 2 & 2 & l & 0.0006 & & & 0.6000 \\
\hline 120 & 2 & 6 & 2 & 1 & 0.0002 & & & 0.3000 \\
\hline 120 & 6 & 2 & 1 & 2 & 0.0003 & & & 0.3000 \\
\hline 120 & 2 & 6 & 1 & l & 0.0004 & & & 0.6000 \\
\hline 120 & 1 & 24 & ] & 1 & 0.0001 & & & 0.6000 \\
\hline 120 & 2 & 6 & 1 & 2 & 0.0001 & & & 0.1000 \\
\hline 120 & 1. & 24 & 1 & 1 & 0.0001 & & & 0.4000 \\
\hline 120 & 1 & 120 & 1 & 1 & 0.0000 & 0.0031 & 0.9994 & $1.0(0)(10)$ \\
\hline 720 & 720 & 1 & 6 & 6 & $0.0000)$ & & & 1.0000 \\
\hline 720 & 120 & 1 & 6 & 2 & 0.0001 & & & 0.5000 \\
\hline 720 & 24 & 2 & 6 & 1 & 0.0000 & & & 0.2000 \\
\hline 720 & 6 & 6 & 6 & 1 & 0.0000 & & & 0.0 .500 \\
\hline 720 & 120 & 1 & 2 & 6 & 0.0001 & & & 0.5000 \\
\hline 720 & 24 & 2 & 2 & 2 & 0.0001 & & & 0.60000 \\
\hline 720 & 6 & 6 & 2 & 1 & 0.0001 & & & 0.4500 \\
\hline 720 & 2 & 24 & 2 & 1 & 0.0000 & & & 0.2000 \\
\hline 720 & 24 & 2 & 1 & 6 & 0.0000 & & & 0.20000 \\
\hline 720 & 6 & 6 & 1 & 2 & 0.0001 & & & 0.4500 \\
\hline 720 & 2 & 24 & 1 & 1 & 0.0000 & & & 0.6000 \\
\hline 720 & 1 & 120 & l & 1. & 0.0000 & & & 0.5000 \\
\hline 720 & 6 & 6 & 1 & 6 & 0,0000 & & & 0.5000 \\
\hline 720 & 2 & 24 & 1 & 2 & 0.0000 & & & 0.2000 \\
\hline 720 & 1 & 120 & l & l & 0,0000 & & & 0.5000 \\
\hline 720 & 1 & 720 & 1 & 1 & 0.0000 & 0.0005 & 0.9999 & 1.0000 \\
\hline 5040 & 5040 & 1 & 24 & 6 & 0.0000 & & & 1.00000 \\
\hline 5040 & 720 & 1 & 24 & 2 & 0.0000 & & & 0.4296 \\
\hline 5040 & 120 & 2 & 24 & 1 & 0.0000 & & & 0.1429 \\
\hline .5040 & 24 & 6 & 24 & 1 & $0.0000)$ & & & 0.0286 \\
\hline 5040 & 720 & 1 & 6 & 6 & 0.0000 & & & $0.5 \div 14$ \\
\hline 5040 & 120 & 2 & 6 & 2 & 0.00000 & & & $0.5 \div 14$ \\
\hline 5040 & 6 & 24 & 6 & 1 & 0.0000 & & & (0.1.143 \\
\hline 5040 & 24 & 6 & 6 & 1 & 0.0000 & & & 0.3429 \\
\hline 5040 & 120 & 2 & 2 & 6 & 0.0000 & & & 0.2857 \\
\hline 5040 & 24 & 6 & 2 & 2 & 0.0000 & & & 0.5143 \\
\hline 5040 & 6 & 24 & 2 & 1 & 0.0000 & & & 0.5143 \\
\hline 5040 & 2 & 120 & 2 & 1 & 0.0000 & & & 0.28 .57 \\
\hline 5040 & 24 & 6 & 1 & 6 & 0.0000 & & & 0.1143 \\
\hline 5040 & 6 & 24 & 1 & 2 & 0.0000 & & & 0.3429 \\
\hline 5040 & 2 & 120 & 1 & 1 & 0.0000 & & & 0.5714 \\
\hline 5040 & 1 & 720 & 1 & 1 & 00000 & & & 0.5714 \\
\hline 5040 & 6 & 24 & 1 & 6 & 0.0000 & & & 0.0286 \\
\hline 5040 & 2 & 120 & 1 & 2 & 0.0000 & & & 0.1429 \\
\hline 5040 & 1 & 720 & 1 & 1 & 0.0000 & & & 0.4286 \\
\hline 5040 & 1 & 5040 & l & 1 & 0.0000 & 0.0001 & 1.0000 & 1.0000 \\
\hline
\end{tabular}


TABLE 4

MARGiNAL PROBABILITY FUNCTION FOR SAMPI.F YFCTORS $\left(n_{n 1}, n_{G 1}\right)$ from table 3

\begin{tabular}{ccccc}
\hline$n$ & $n_{R 1}$ & $n_{G 1}$ & $p\left(n_{R 1}, n_{G 1}\right)$ & Cimulative \\
\hline 0 & 0 & 0 & 0.5315 & 0.5315 \\
1 & 1 & 0 & 0.2869 & 0.8184 \\
1 & 0 & 1 & 0.0491 & 0.8675 \\
2 & 1 & 1 & 0.0952 & 0.9627 \\
2 & 2 & 0 & 0.0100 & 0.9727 \\
2 & 0 & 2 & 0.0009 & 0.9736 \\
3 & 3 & 0 & 0.0001 & 0.9737 \\
3 & 2 & 1 & 0.0190 & 0.9927 \\
3 & 1 & 2 & 0.0032 & 0.9959 \\
3 & 0 & 3 & 0.0000 & 0.9959 \\
4 & 4 & 0 & 0.0000 & 0.9959 \\
4 & 3 & 1 & 0.0004 & 0.9963 \\
4 & 2 & 2 & 0.0030 & 0.9993 \\
4 & 1 & 3 & 0.0000 & 0.9993 \\
4 & 0 & 4 & 0.0000 & 0.9993 \\
5 & 5 & 0 & 0.0000 & 0.9993 \\
5 & 4 & 1 & 0.0000 & 0.9993 \\
5 & 3 & 2 & 0.0004 & 0.9997 \\
5 & 2 & 3 & 0.0001 & 0.9998 \\
5 & 1 & 4 & 0.0000 & 0.9998 \\
5 & 0 & 5 & 0.0000 & 0.9998 \\
\hline
\end{tabular}

in Table 5 is similarly obtained from Table 3. The marginal distribution of count of red (R) units in node 1 shown in Table 6 can be obtained from either Tables 3, 4, or 5 although roundoff errors will vary.

Table 6 shows the distribution of the count of red units in node 1 when the arrival process of red units is Camma distributed. If, for example, node 1 is a multi-station service facility for arriving units where interarrival times are independent and Camma distributed, Table 6 gives the distribution of the number of incomplete services at time $t=1$, the mean number at that time being 0.452 .

Table 3 is a direct printout of a spreadsheet in which certain columns of intermediate calculations are hidden. A data base tool for generating sample vectors and their joint probabilities could have been used as well. Commercial databases have very large capacities for records (sample vectors) so the issue is whether speed of computation is sufficient to permit this method to be practical for systems involving numbers of sample vectors on the order of millions. 
TABLE 5

MARGINAL PROBABILITY FUNCTION FOR SAMPLE IECTORS $\left(n_{k 1}, n_{R 2}\right)$ FROM TABIE 3

\begin{tabular}{cccc}
\hline$n_{R 1}$ & $n_{R 2}$ & $p\left(n_{R 1}, n_{R 2}\right)$ & Cumulative \\
\hline 0 & 0 & 0.3679 & 0.3679 \\
1 & 0 & 0.3488 & 0.7167 \\
0 & 1 & 0.2030 & 0.9197 \\
2 & 0 & 0.0305 & 0.9502 \\
1 & 1 & 0.0356 & 0.9858 \\
0 & 2 & 0.0104 & 0.9962 \\
3 & 0 & 0.0009 & 0.9971 \\
2 & 1 & 0.0016 & 0.9987 \\
1 & 2 & 0.0009 & 0.9996 \\
0 & 3 & 0.0002 & 0.9998 \\
4 & 0 & 0.0000 & 0.9998 \\
3 & 1 & 0.0000 & 0.9998 \\
2 & 2 & 0.0000 & 0.9998 \\
1 & 3 & 0.0000 & 0.9998 \\
0 & 4 & 0.0000 & 0.9998 \\
\hline
\end{tabular}

TABLE 6

MARGINAL PROBABILITY FUNCTION FOR SAMPLE POINTS $\left(n_{R 1}\right)$ FROM TABLE 3

\begin{tabular}{ccc}
\hline$n_{R 1}$ & $p\left(n_{R 1}\right)$ & Cumulative \\
\hline 0 & 0.5815 & 0.5815 \\
1 & 0.3853 & 0.9668 \\
2 & 0.0321 & 0.9989 \\
3 & 0.0009 & 0.9998 \\
4 & 0.0000 & 0.9998 \\
\hline
\end{tabular}

\section{CONCLUSION}

Small systems in the family $(\pi(D), \Sigma(K))$ are easily modelled using spreadsheet or database software subject to the condition that interval transition probability functions can be computed. The problem of computing interval probability functions is not discussed in this paper. Exact representations of interval transition probability functions are not feasible in the general case although they are available for many networks with simplified connective topology.

Non-Poisson arrival processes, including processes in which interarrival time intervals are not identically distributed, are easily represented by insert- 
ing units carrying tags of other colors into the arrival stream to serve as position markers. The price of this generality is an increase in the number of sample vectors that must be included when spreadsheet or database methods are employed for computation.

Populations of units arriving at random in time, colors of arrivals being mixed in a known order, represent a generic model interpretable in many disciplinary contexts including biology, engineering, health sciences, demography, and operations research. 